\title{
PICTURE FUZZY SETS
}

\author{
BUI CONG CUONG \\ Institute of Mathematics, Vietnam Academy of Science and Technology \\ bccuong@gmail.com
}

\begin{abstract}
In this paper, we introduce the concept of picture fuzzy sets (PFS), which are direct extensions of the fuzzy sets and the intuitonistic fuzzy sets. Then some operations on PFS with some properties are considered. The following sections are devoted to the Zadeh Extension Principle, picture fuzzy relations and picture fuzzy soft sets. Here, the basic preliminaries of PFS theory are presented.
\end{abstract}

Keywords. Picture fuzzy set, operation, picture fuzzy relation, picture fuzzy soft set.

\section{INTRODUCTION}

In this section we propose the definition of picture fuzzy sets and some operators on PFS.

Definition 1.1. A picture fuzzy set $A$ on a universe $X$ is an object in the form of

$$
A=\left\{\left(x, \mu_{A}(x), \eta_{A}(x), \nu_{A}(x)\right) \mid x \in X\right\}
$$

where $\mu_{A}(x) \in[0,1]$ is called the degree of positive membership of $x$ in $A, \eta_{A}(x) \in[0,1]$ is called the degree of neutral membership of $x$ in $A$ and $\nu_{A}(x) \in[0,1]$ is called the degree of negative membership of $x$ in $A$, and where $\mu_{A}, \eta_{A}$ and $\nu_{A}$ satisfy the following condition:

$$
(\forall x \in X) \quad\left(\mu_{A}(x)+\eta_{A}(x)+\nu_{A}(x) \leq 1\right)
$$

Now $\left(1-\left(\mu_{A}(x)+\eta_{A}(x)+\nu_{A}(x)\right)\right.$ could be called the degree of refusal membership of $x$ in $A$. Let $P F S(X)$ denote the set of all the picture fuzzy sets on a universe $X$.

Basically, picture fuzzy sets based models may be adequate in situations when we face human opinions involving more answers of types: yes, abstain, no, refusal. Voting can be a good example of such a situation as the human voters may be divided into four groups of those who: vote for, abstain, vote against, refusal of the voting. PFS is a direct generalization of the fuzzy set was introduced by Zadeh 1965 [16] and the intuitionistic fuzzy set was proposed by Atanassov 1983 [3].

Definition 1.2 ( [3]) A intuitionistic fuzzy set $A$ on a universe $X$ is an object of the form

$$
A=\left\{\left(x, \mu_{A}(x), \nu_{A}(x) \mid x \in X\right)\right\}
$$

where $\mu_{A}(x) \in[0,1]$ is called the degree of membership of $x$ in $A, \nu_{A}(u) \in[0,1]$ is called the degree of non-membership of $x$ in $A$, and where $\mu_{A}$ and $\nu_{A}$ satisfy the following condition:

$$
(\forall x \in X) \quad\left(\mu_{A}(x)+\nu_{A}(x) \leq 1\right)
$$

In this paper, let $I F S(X)$ denote the set of all the intuitionistic fuzzy sets (IFSs) on a universe $X$.

(c) 2014 Vietnam Academy of Science \& Technology 
Definition 1.3 ( [10]) For every two PFSs $A$ and $B$, the union, intersection and complement are defined as follows:

- $A \subseteq B$ iff $\left(\forall x \in X, \mu_{A}(x) \leq \mu_{B}(x)\right.$ and $\eta_{A}(x) \leq \eta_{B}(x)$ and $\left.\nu_{A}(x) \geq \nu_{B}(x)\right)$

- $A=B \quad \operatorname{iff}(A \subseteq B$ and $B \subseteq A)$

- $A \cup B=\left\{\left(x, \max \left(\mu_{A}(x), \mu_{B}(x)\right), \min \left(\eta_{A}(x), \eta_{B}(x)\right), \min \left(\nu_{A}(x), \nu_{B}(x)\right) \mid x \in X\right\}\right.$

- $A \cap B=\left\{\left(x, \min \left(\mu_{A}(x), \mu_{B}(x)\right), \min \left(\eta_{A}(x), \eta_{B}(x)\right), \max \left(\nu_{A}(x), \nu_{B}(x)\right) \mid x \in X\right\}\right.$

- $\operatorname{co}(A)=\bar{A}=\left\{\left(\nu_{A}(x), \eta_{A}(x), \mu_{A}(x)\right) \mid x \in X\right\}$

Now, a generalization of interval-valued fuzzy set $A$ is proposed. Here the int $([0,1])$ stands for the set of all closed subinterval of $[0,1]$.

Definition 1.4 ( $[6])$ Let $\left[a_{1}, b_{1}\right],\left[a_{2}, b_{2}\right] \in \operatorname{int}([0,1])$. We define

$$
\begin{array}{lll}
{\left[a_{1}, b_{1}\right] \leq\left[a_{2}, b_{2}\right],} & \text { iff } a_{1} \leq a_{2}, b_{1} \leq b_{2} ;\left[a_{1}, b_{1}\right] \precsim\left[a_{2}, b_{2}\right] & \text { iff } a_{1} \leq a_{2}, b_{1} \geq b_{2} ; \\
{\left[a_{1}, b_{1}\right]=\left[a_{2}, b_{2}\right],} & \text { iff } a_{1}=a_{2}, b_{1}=b_{2} .
\end{array}
$$

Definition 1.5. An interval-valued picture fuzzy set $A$ on a universe $X$ (IvPFS, in short) is an object of the form

$$
A=\left\{\left(x, M_{A}(x), L_{A}(x), N_{A}(x)\right) \mid x \in X\right\}
$$

where

$$
\begin{aligned}
& M_{A}: X \rightarrow \operatorname{int}([0,1]), M_{A}(x)=\left[M_{A L}(x), M_{A U}(x)\right] \in \operatorname{int}([0,1]) \\
& L_{A}: X \rightarrow \operatorname{int}([0,1]), L_{A}(x)=\left[L_{A L}(x), L_{A U}(x)\right] \in \operatorname{int}([0,1]) \\
& N_{A}: X \rightarrow \operatorname{int}([0,1]), N_{A}(x)=\left[N_{A L}(x), N_{A U}(x)\right] \in \operatorname{int}([0,1])
\end{aligned}
$$

satisfy the following condition:

$$
(\forall x \in X) \quad\left(\sup M_{A}(x)+\sup L_{A}(x)+\sup N_{A}(x) \leq 1\right)
$$

Let $\operatorname{IvPFS}(X)$ denote the set of all the interval-valued picture fuzzy set IvPFSs on a universe $X$.

Definition 1.6. For every two IvPFSs A and B, the inclusion, union, intersection and complement are defined as follows:

$A \subseteq B$ iff $(\forall x \in X)\left(M_{A L}(x) \leq M_{B L}(x)\right.$ and $\left.M_{A U}(x) \leq M_{B U}(x)\right)$ and

- $\left(L_{A L}(x) \leq L_{B L}(x)\right.$ and $\left.L_{A U}(x) \leq L_{B U}(x)\right)$ and $\left(N_{A L}(x) \geq N_{B L}(x)\right.$ and $\left.\left.N_{A U}(x) \geq N_{B U}(x)\right]\right)$.

- $A=B$ iff $A \subseteq B$ and $B \subseteq A$

- $A \cup B=\left\{\left(x,\left[M_{A L}(x) \vee M_{B L}(x), M_{A U}(x) \vee M_{B U}(x)\right]\right.\right.$,

- $\left.\left.\left[L_{A L}(x) \wedge L_{B L}(x), L_{A U}(x) \wedge L_{B U}(x)\right],\left[N_{A L}(x) \wedge N_{B L}(x), N_{A U}(x) \wedge N_{B U}(x)\right]\right) \mid x \in X\right\}$

- $A \cap B=\left\{\left(x,\left(\left[M_{A L}(x) \wedge M_{B L}(x), M_{A U}(x) \wedge M_{B U}(x)\right]\right.\right.\right.$,

- $\left.\left.\left[L_{A L}(x) \wedge L_{B L}(x), L_{A U}(x) \wedge L_{B U}(x)\right],\left[N_{A L}(x) \vee N_{B L}(x), N_{A U}(x) \vee N_{B U}(x)\right]\right) \mid x \in X\right\}$ 
where $\vee$ and $\wedge$ stand for max and min operators respectively

$$
\operatorname{co} A=\bar{A}=\left\{\left(x, N_{A}(x), L_{A}(x), M_{A}(x)\right) \mid x \in X\right\}
$$

Definition 1.7. Let $X_{1}$ and $X_{1}$ be two universes and let

$$
A=\left\{\left(x, \mu_{A}(x), \eta_{A}(x), \nu_{A}(x)\right) \mid x \in X_{1}\right\} \text { and } B=\left\{\left(y, \mu_{B}(y), \eta_{B}(y), \nu_{B}(y)\right) \mid y \in X_{2}\right\}
$$

be two PFSs. The Cartesian product of these two PFSs is defined as follows:

- $A \times_{1} B=\left\{\left((x, y), \mu_{A}(x) \cdot \mu_{B}(y), \eta_{A}(x) \cdot \eta_{B}(y), \nu_{A}(x) \cdot \nu_{B}(y)\right) \mid x \in X_{1}, y \in X_{2}\right\}$

- $A \times_{2} B=\left\{\left((x, y), \mu_{A}(x) \wedge \mu_{B}(y), \eta_{A}(x) \wedge \eta_{B}(y), \nu_{A}(x) \vee \nu_{B}(y)\right) \mid x \in X_{1}, y \in X_{2}\right\}$

These definitions are valid. See $[7,8]$.

Definition 1.8. Let $A$ be an IvPFS over $X_{1}$ and $B$ be an IvPFS over $X_{2}$ We define:

- $A \times_{1} B=\left\{(x, y),\left(\left[M_{A L}(x) \cdot M_{B L}(y), M_{A U}(x) \cdot M_{B U}(y)\right]\right.\right.$

- $\left.\left.\left[L_{A L}(x) \cdot L_{B L}(y), L_{A U}(x) \cdot L_{B U}(y)\right],\left[N_{A L}(x) \cdot N_{B L}(y), N_{A U}(x) \cdot N_{B U}(y)\right]\right) \mid x \in X_{1}, y \in X_{2}\right\}$

- $A \times_{2} B=\left\{(x, y),\left(\left[M_{A L}(x) \wedge M_{B L}(y), M_{A U}(x) \wedge M_{B U}(y)\right]\right.\right.$,

- $\left.\left.\left[L_{A L}(x) \wedge L_{B L}(y), L_{A U}(x) \wedge L_{B U}(y)\right],\left[N_{A L}(x) \vee N_{B L}(y), N_{A U}(x) \vee N_{B U}(y)\right]\right) \mid x \in X_{1}, y \in X_{2}\right\}$

The defined operations are extensions of the operations on FSs given in $[11,14,16]$ and of the operations for IFSs and IVIFSs given in [2-6].

\section{SOME OPERATIONS ON PFS}

Now, some properties of the defined operations on PFSs are considered.

\subsection{Some propositions}

Proposition 2.1. For every PFSs $A, B, C$

(a) If $A \subseteq B$ and $B \subseteq C$ then $A \subseteq C$;

(b) Operations $\cap$ and $\cup$ are commutative;

(c) Operations $\cap$ and $\cup$ are associative;

(c) Operations $\cap$ and $\cup$ are distributive;

(c) Operations $\cap$ Co and $\cup$ are satisfy the law of De Morgan.

Now we present some properties of the Cartesian product of two PFSs.

Definition 2.1. Let $X_{1}$ and $X_{2}$ be two universes. Let $A \in \operatorname{IvPFS}\left(X_{1}\right), B \in \operatorname{IvPFS}\left(X_{2}\right)$. Then

$A \times_{2} B=\left\{\left((x, y),\left[M_{A L}(x) \wedge M_{B L}(y), M_{A U}(x) \wedge M_{B U}(y)\right]\right.\right.$, $\left.\left.\left[L_{A L}(x) \wedge L_{B L}(y), L_{A U}(x) \wedge L_{B U}(y)\right],\left[N_{A L}(x) \vee N_{B L}(y), N_{A U}(x) \vee N_{B U}(y)\right]\right) \mid x \in X_{1}, y \in X_{2}\right\}$.

Proposition 2.2. For every three universes $X_{1}, X_{2}, X_{3}$ and four PFSs $A, B \in P F S\left(X_{1}\right)$, $C \in P F S\left(X_{2}\right), D \in P F S\left(X_{3}\right)$ : 

(a) $A \times{ }_{2} C=C \times{ }_{2} A$
(b) $\left(A \times{ }_{2} C\right) \times{ }_{2} D=A \times{ }_{2}\left(C \times{ }_{2} D\right)$
(c) $(A \cup B) \times{ }_{2} C=\left(A \times{ }_{2} C\right) \cup\left(B \times{ }_{2} C\right)$
(d) $(A \cap B) \times{ }_{2} C=\left(A \times{ }_{2} C\right) \cap\left(B \times{ }_{2} C\right)$

Proof. Obvious.

\subsection{Distance between picture fuzzy sets}

The following definition is an extension of the distances between intuitionistic fuzzy sets given by Szmidt and Kacprzyk in [15].

Definition 2.2. Distances for two picture fuzzy sets $A$ and $B$ in $X=\left\{x_{1}, x_{2}, \ldots, x_{n}\right\}$ are:

- The normalized Hamming distance $d_{P}(A, B)$

$$
d_{P}(A, B)=\frac{1}{n} \sum_{i=1}^{n}\left(\left|\mu_{A}\left(x_{i}\right)-\mu_{B}\left(x_{i}\right)\right|+\left|\eta_{A}\left(x_{i}\right)-\eta_{B}\left(x_{i}\right)\right|+\left|\nu_{A}\left(x_{i}\right)-\nu_{B}\left(x_{i}\right)\right|\right.
$$

- The normalized Euclidean distance $e_{P}(A, B)$

$$
e_{P}(A, B)=\sqrt{\left.\frac{1}{n} \sum_{i=1}^{n}\left(\left(\mu_{A}\left(x_{i}\right)\right)-\mu_{B}\left(x_{i}\right)\right)^{2}+\left(\eta_{A}\left(x_{i}\right)-\eta_{B}\left(x_{i}\right)\right)^{2}+\left(\nu_{A}\left(x_{i}\right)-\nu_{B}\left(x_{i}\right)\right)^{2}\right)}
$$

Example 2.1. Let us consider picture fuzzy sets $A, B, C$ in $X=\left\{x_{1}, x_{2}, x_{3}\right\}$, The full description of picture fuzzy set $A$, i.e.

$A=\left\{\left(\mu_{A}\left(x_{1}\right), \eta_{A}\left(x_{1}\right), \nu_{A}\left(x_{1}\right)\right) / x_{1},\left(\mu_{A}\left(x_{2}\right), \eta_{A}\left(x_{2}\right), \nu_{A}\left(x_{2}\right)\right) / x_{2},\left(\mu_{A}\left(x_{3}\right), \eta_{A}\left(x_{3}\right), \nu_{A}\left(x_{3}\right)\right) / x_{3}\right\}$

For example,

$$
\begin{aligned}
& A=\left\{(0.8,0.1,0) / x_{1},(0.4,0.2,0.3) / x_{2},(0.5,0.3,0) / x_{3}\right\} \\
& B=\left\{(0.3,0.3,0.2) / x_{1},(0.7,0.1,0.1) / x_{2},(0.4,0.3,0.2) / x_{3}\right\} \\
& C=\left\{(0.3,0.4,0.1) / x_{1},(0.6,0.2,0.1) / x_{2},(0.4,0.3,0.1) / x_{3}\right\}
\end{aligned}
$$

Then

$$
d_{P}(A, B)=0.6, d_{P}(B, C)=0.5 / 3, d_{P}(A, C)=0.5
$$

and

$$
e_{P}(A, B)=\sqrt{0.52 / 3}, e_{P}(B, C)=\sqrt{0.05 / 3}, e_{P}(A, C)=\sqrt{0.15}
$$

\subsection{Convex combination of PFS}

Convex combination is an important operation in mathematics, which is an useful tool on convex analysis, linear spaces and convex optimization. In this section, convex combination of PFSs firstly is defined with some simple propositions. 
Definition 2.3. Let $A, B$, be a PFS on $X$. Let $\theta$ be a real number such that $0 \leq \theta \leq 1$. For each $\theta$, the convex combination of $A$ and $B$ is defined as follows:

$C_{\theta}(A, B)=\left\{\left(\left(x, \mu_{C_{\theta}}(x), \eta_{C_{\theta}}(x), \nu_{C_{\theta}}(x)\right) \mid x \in X\right)\right\}$ where

$\forall x \in X, \mu_{C_{\theta}}(x)=\theta \cdot \mu_{A}(x)+(1-\theta) \mu_{B}(x)$

$\forall x \in X, \eta_{C_{\theta}}(x)=\theta \cdot \eta_{A}(x)+(1-\theta) \eta_{B}(x), \forall x \in X, \nu_{C_{\theta}}(x)=\theta \cdot \nu_{A}(x)+(1-\theta) \nu_{B}(x)$

Definition 2.4. Let $A, B$ be a PFS on $X$. Let $\theta$ be a real number $0 \leq \theta \leq 1$. Then

- If $\theta=1$, then $C_{\theta}(A, B)=A$ and if $\theta=0$, then $C_{\theta}(A, B)=B$;

- $A \subseteq B$ then $\forall \theta, \quad A \subseteq C_{\theta}(A, B) \subseteq B$

- $(A \supseteq B) \&\left(\theta_{1} \geq \theta_{2}\right)$ then $C_{\theta_{1}}(A, B) \supseteq C_{\theta_{2}}(A, B)$

Proof. The proof is immediate.

Proposition 2.3. Let $A, B \in P F S(X)$. Let $\theta$ be a real number such that $0 \leq \theta \leq 1$. Then

- $C_{\theta}(A \cap B, D)=C_{\theta}(A, D) \cap C_{\theta}(B, D)$

- $C_{\theta}(A \cup B, D)=C_{\theta}(A, D) \cup C_{\theta}(B, D)$

Proof. See [8].

\subsection{More on interval valued PFS}

Some selected operations for interval valued picture fuzzy sets are considered: the convex combination of IvPFSs and the Cartesian product of IvPFSs.

Definition 2.5. Let $A, B$ be two IvPFS on $X$. Let $\theta$ be a real number, such is $0 \leq \theta \leq 1$. For each $\theta$, the convex combination of $A$ and $B$ is defined as follows:

$$
C_{\theta}(A, B)=\left\{\left(\left(x, M_{C_{\theta}}(x), L_{C_{\theta}}(x), N_{C_{\theta}}(x)\right) \mid x \in X\right)\right\}
$$

where

$\forall x \in X, M_{C_{\theta}}(x)=\left[\left(M_{C_{\theta} L}(x)=\theta \cdot M_{A L}(x)+(1-\theta) \cdot M_{B L}(x)\right),\left(M_{C_{\theta} U}(x)=\theta \cdot M_{A U}(x)+(1-\theta) M_{B U}(x)\right)\right]$, $\forall x \in X, L_{C_{\theta}}(x)=\left[\left(L_{C_{\theta} L}(x)=\theta \cdot L_{A L}(x)+(1-\theta) \cdot L_{B L}(x)\right),\left(L_{C_{\theta} U}(x)=\theta \cdot L_{A U}(x)+(1-\theta) L_{B U}(x)\right)\right]$, $\forall x \in X, N_{C_{\theta}}(x)=\left[\left(N_{C_{\theta} L}(x)=\theta \cdot N_{A L}(x)+(1-\theta) \cdot N_{B L}(x)\right),\left(N_{C_{\theta} U}(x)=\theta \cdot N_{A U}(x)+(1-\theta) \cdot N_{B U}(x)\right)\right]$.

Proposition 2.4. Let $A, B$, be two IvPFSs on $X$. Let $\theta$ be a real number $0 \leq \theta \leq 1$, then

- If $\theta=1$ then $C_{\theta}(A, B)=A$ and if $\theta=0$, then $C_{\theta}(A, B)=B$;

- If $A \subseteq B$ then $\forall \theta, \quad A \subseteq C_{\theta}(A, B) \subseteq B$

- If $(A \supseteq B) \&\left(\theta_{1} \geq \theta_{2}\right)$, then $C_{\theta_{1}}(A, B) \supseteq C_{\theta_{2}}(A, B)$

Proof. Proof. Immediate.

Proposition 2.5. Let $A, B, D \in \operatorname{IvPFS}(X)$. Let $\theta$ be a real number such that $0 \leq \theta \leq 1$. Then

- $C_{\theta}(A \cap B, D)=C_{\theta}(A, D) \cap C_{\theta}(B, D)$

- $C_{\theta}(A \cup B, D)=C_{\theta}(A, D) \cup C_{\theta}(B, D)$

Proof. Immediate.

Some propositions of the Cartesian product of two PFSs and two IvPFSs are given in $[7,8,10]$. 


\section{PICTURE FUZZY RELATIONS}

Fuzzy relations are ones of most important notions of fuzzy set theory and fuzzy systems theory. The Zadeh composition rule of fuzzy relations in inference procedures (see $[14,16]$ ) is a well-known method in approximation theory and in the fuzzy control theory. Then intuitionistic fuzzy relations were defined, many results were obtained by researches (see $[5,6])$. In this section, some preliminary results on picture fuzzy relations are presented.

\subsection{Some definitions}

Let $X, Y$ and $Z$ be ordinary non-empty sets.

An extension result of the given proposition in $[5,6]$ for PFS is as follows

Definition 3.1. A picture fuzzy relation is a picture fuzzy subset of $X \times Y$ i.e. $R$ given by

$$
\left.R=\left\{\left((x, y), \mu_{R}(x, y), \eta_{R}(x, y), \nu_{R}(x, y)\right) \mid x \in X, y \in Y\right)\right\}
$$

where $\mu_{R}: X \times Y \rightarrow[0,1], \eta_{R}: X \times Y \rightarrow[0,1], \nu_{R}: X \times Y \rightarrow[0,1]$ satisfy the condition $0 \leq \mu_{R}(x, y)+\eta_{R}(x, y)+\nu_{R}(x, y) \leq 1$ for every $(x, y) \in(X \times Y)$. PFR $(X \times Y)$ the set of all the picture fuzzy relations in $X \times Y$ is denoted.

Definition 3.2. Let $R \in P F R(X \times Y)$. We define the inverse relation $R^{-1}$ between $Y$ and $X: \mu_{R^{-1}}(y, x)=\mu_{R}(x, y), \eta_{R^{-1}}(y, x)=\eta_{R}(x, y), \nu_{R^{-1}}(y, x)=\nu_{R}(x, y), \forall(x, y) \in(X \times Y)$ Some properties of PFRs are the followings:

Definition 3.3. Let $R$ and $P$ be two picture fuzzy relations between $X$ and $Y$, for every $(x, y) \in X \times Y$ we define:

- $R \leq P \Leftrightarrow\left(\mu_{R}(x, y) \leq \mu_{P}(x, y)\right) \&\left(\eta_{R}(x, y) \leq \eta_{P}(x, y)\right) \&\left(\nu_{R}(x, y) \geq \nu_{P}(x, y)\right)$

- $R \vee P=\left\{\left((x, y), \mu_{R}(x, y) \vee \mu_{P}(x, y), \eta_{R}(x, y) \wedge \eta_{P}(x, y), \nu_{R}(x, y) \wedge \nu_{P}(x, y)\right) \mid x \in X, y \in Y\right\}$

- $R \wedge P=\left\{\left((x, y), \mu_{R}(x, y) \wedge \mu_{P}(x, y), \eta_{R}(x, y) \wedge \eta_{P}(x, y), \nu_{R}(x, y) \vee \nu_{P}(x, y)\right) \mid x \in X, y \in Y\right\}$

- $R_{c}=\left\{\left((x, y), \nu_{R}(x, y), \eta_{R}(x, y), \mu_{R}(x, y)\right) \mid x \in X, y \in Y\right\}$

Proposition 3.1. Let $R, P, Q \in P F R(X \times Y)$. Then

(a) $\left(R^{-1}\right)^{-1}=R$

(b) $R \leq P \Rightarrow R^{-1} \leq P^{-1}$

(c1) $(R \vee P)^{-1}=R^{-1} \vee P^{-1}$

(c2) $(R \wedge P)^{-1}=R^{-1} \wedge P^{-1}$

(d1) $R \wedge(P \vee Q)=(R \wedge P) \vee(R \wedge Q)$

$(\mathrm{d} 2) R \vee(P \wedge Q)=(R \vee P) \wedge(R \vee Q)$

(e) $R \wedge P \leq R, R \wedge P \leq P$

(f1) If $(R \geq P) \&(R \geq Q)$ then $R \geq P \vee Q$

(f2) If $(R \leq P) \&(R \leq Q)$ then $R \leq P \wedge Q$

Proof. See $[7,8]$. 


\subsection{Composition of Picture Fuzzy Relations}

We know that the composition of intuitionistic fuzzy relations is given in [5] as follows:

Definition 3.4 ( [5]) Let $\alpha, \beta, \lambda, \rho$ be $t$-norms or $t$-conorms not necessarily dual two-two, $E \in \operatorname{IFR}(X \times Y)$ and $P \in \operatorname{IFR}(Y \times Z)$. Composed relation $P \mathcal{C E} \in \operatorname{IFR}(X \times Z)$ is called to the one defined by

$$
\left.P \mathcal{C} \mathcal{E}=\left\{\left((x, z), \mu_{P \mathcal{E}}(x, z), \nu_{P E}(x, z)\right) \mid x \in X, z \in Z\right)\right\}
$$

with

$$
\mu_{P \mathcal{C E}}(x, z)=\underset{y}{\alpha}\left\{\beta\left[\mu_{E}(x, y), \mu_{P}(y, z)\right]\right\}, \nu_{P \mathcal{C}}(x, z)=\underset{y}{\lambda}\left\{\rho\left[\nu_{E}(x, y), \nu_{P}(y, z)\right]\right\}
$$

whenever

$$
0 \leq \mu_{P \mathcal{C E}}(x, z)+\nu_{P \mathcal{C E}}(x, z) \leq 1 \quad \forall(x, z) \in X \times Z
$$

In [5] it was proved that if $\alpha=\vee, \beta t$-norm $\lambda=\wedge, \rho t-$ conorm, the composition of intuitionistic fuzzy relations satisfies many properties.

A composition of picture fuzzy relations is the following:

Definition 3.5. Let $\alpha=\vee, \beta t$-norm , $\lambda=\wedge, \rho t-$ conorm, $E \in P F R(X \times Y)$ and $P \in P F R(Y \times Z)$. Composed relation $P C E \in P F R(X \times Z)$ is called to the one defined by

$$
\left.P \mathcal{C E}=\left\{\left((x, z), \mu_{P \mathcal{C E}}(x, z), \eta_{P \mathcal{C E}}(x, z), \nu_{P \mathcal{C E}}(x, z)\right) \mid x \in X, z \in Z\right)\right\},
$$

where

$$
\begin{aligned}
\mu_{P \mathcal{E}}(x, z) & ={\underset{y}{\vee}}_{y}\left\{\beta\left[\mu_{E}(x, y), \mu_{P}(y, z)\right]\right\} \\
\eta_{P \mathcal{E}}(x, z) & =\bigwedge_{y}\left\{\beta\left[\eta_{E}(x, y), \eta_{P}(y, z)\right]\right\} \\
\nu_{P \mathcal{C E}}(x, z) & =\bigwedge_{y}\left\{\rho\left[\nu_{E}(x, y), \nu_{P}(y, z)\right]\right\}
\end{aligned}
$$

whenever

$$
0 \leq \mu_{P \mathcal{C E}}(x, z)+\eta_{P \mathcal{C E}}(x, z)+\nu_{P \mathcal{C E}}(x, z) \leq 1 \quad \forall(x, z) \in X \times Z
$$

The following composition of PFRs is the generalized min-max composition of fuzzy relations.

Definition 3.6. Let $E \in P F R(X \times Y)$ and $P \in P F R(Y \times Z)$. Max-min composed relation $P C E \in P F R(X \times Z)$ is called to the one defined by

$$
\left.P \mathcal{C E}=\left\{\left((x, z), \mu_{P \mathcal{C E}}(x, z), \eta_{P \mathcal{C E}}(x, z), \nu_{P \mathcal{C E}}(x, z)\right) \mid x \in X, z \in Z\right)\right\}
$$

where $\forall(x, z) \in X \times Z, \mu_{P \mathcal{C E}}(x, z)={\underset{y}{V}}_{y}\left\{\left[\mu_{E}(x, y) \wedge \mu_{P}(y, z)\right]\right\}$

$$
\begin{aligned}
\eta_{P \mathcal{E}}(x, z) & ={\underset{y}{\wedge}}_{\wedge}\left\{\left[\eta_{E}(x, y) \wedge \eta_{P}(y, z)\right]\right\} \\
\nu_{P \mathcal{E}}(x, z) & ={\underset{y}{\wedge}}_{\wedge}\left\{\left[\nu_{E}(x, y) \vee \nu_{P}(y, z)\right]\right\}
\end{aligned}
$$

The second composition of PFRs is the generalized min-prod composition in fuzzy set theory: 
Definition 3.7. Let $E \in P F R(X \times Y)$ and $P \in P F R(Y \times Z)$. Let $t_{1}, t_{2}$ be $t$-norms . Max-t-norms composed relation $P \mathcal{C E} \in P F R(X \times Z)$ is called to the one defined by

$$
\left.P \mathcal{C E}=\left\{\left((x, z), \mu_{P \mathcal{C E}}(x, z), \eta_{P \mathcal{C E}}(x, z), \nu_{P E}(x, z)\right) \mid x \in X, z \in Z\right)\right\}
$$

where $\forall(x, z) \in X \times Z$,

$$
\begin{gathered}
\mu_{P \mathcal{C E}}(x, z)=\underset{y}{\vee}\left\{t_{1}\left(\mu_{E}(x, y), \mu_{P}(y, z)\right)\right\} \\
\eta_{P \mathcal{C E}}(x, z)=\bigwedge_{y}\left\{t_{2}\left(\eta_{E}(x, y), \eta_{P}(y, z)\right)\right\} \\
\nu_{P \mathcal{C E}}(x, z)=\bigwedge_{y}\left\{\left[\nu_{E}(x, y)+\nu_{P}(y, z)-\nu_{E}(x, y) \cdot \nu_{P}(y, z)\right]\right\}
\end{gathered}
$$

\section{ZADEH EXTENSION PRINCIPLE FOR PFS}

The Zadeh Extension Principle is an important tool for many problems of fuzzy set theory, fuzzy control and applications. Now a version of the Zadeh Extension Principle for PFS should be presented. The following simple proposition is considered first.

Let $X_{1}$ and $X_{2}$ be two universes. Let $A=\left\{\left(x, \mu_{A}(x), \eta_{A}(x), \nu_{A}(x)\right) \mid x \in X_{1}\right\}$ and $B=\left\{\left(y, \mu_{B}(y), \eta_{B}(y), \nu_{B}(y)\right) \mid y \in X_{2}\right\}$ be two PFSs. The following Cartesian product of PFSs is considered:

$$
A \times_{2} B=\left\{\left((x, y), \mu_{A}(x) \wedge \mu_{B}(y), \eta_{A}(x) \wedge \eta_{B}(y), \nu_{A}(x) \vee \nu_{B}(y)\right) \mid x \in X_{1}, y \in X_{2}\right\} .
$$

Proposition 4.1. Let for $i=1,2, \cdots, n, X_{i}$ be a universe and $A_{i}=\left\{\left(x, \mu_{A_{i}}(x), \eta_{A_{i}}(x)\right.\right.$, $\left.\left.\nu_{A_{i}}(x)\right) \mid x \in X_{i}\right\}$ be a PFS on $X_{i}$ Then, the Cartesian product of PFSs

$$
B^{n}=\stackrel{X}{X}_{i=1}^{n} A_{i}=\left\{\left(\left(x_{1}, . ., x_{n}\right), \wedge_{i=1}^{n} \mu_{A_{i}}\left(x_{i}\right), \stackrel{\wedge}{n}_{i=1}^{n} \eta_{A_{i}}\left(x_{i}\right), \underset{i=1}{\vee n} \nu_{A_{i}}(x)\right) \mid \forall x_{i} \in X_{i}, i=1, \cdots, n\right\}
$$

is a PFS on $\underset{i=1}{X} X_{i}$

Proof. Proof. We prove by inductive reasoning. For $n=2$, the result is given in the Definition 2.1. Now by inductive reasoning $B^{n-1}=\underset{i=1}{X} A_{i}$ is a PFS on $\underset{i=1}{X} X_{i}$, hence $B^{n}=B^{n-1} \times_{2} A_{n}=\underset{i=1}{\underset{X}{X}} A_{i}$ is a PFS of $\underset{i=1}{X} X_{i}$

Proposition 4.2. The Zadeh Extension Principle for PFS. Let for $i=1,2, \cdots, n, U_{i}$ be a universe and let $V \neq \emptyset$. Let $f: \stackrel{n}{X} U_{i} \rightarrow V$ be a mapping, where $y=f\left(z_{1}, \cdots, z_{n}\right)$. Let $z_{i}$ is a linguistic variable on $U_{i}$ for $i=1,2, \cdots, n$. Suppose, for all $i, z_{i}$ is $A_{i}$, where $A_{i}$ is a PFS on $U_{i}$, then the output of the mapping $f$ is $B$. The set $B$ is a PFS on $V$ defined for $y \in V$ by

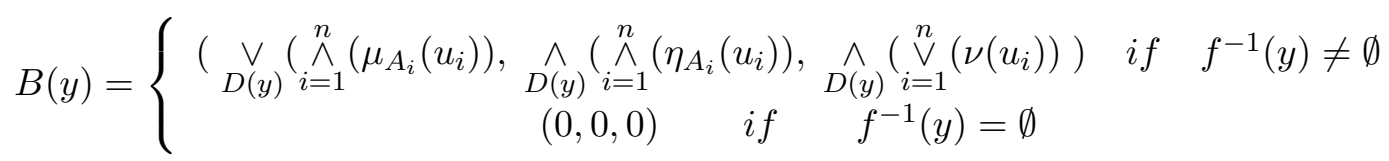

where $D(y)=f^{-1}(y)=\left\{u=\left(u_{1}, \cdots, u_{n}\right): f(u)=y\right\}$ 


\section{PICTURE FUZZY SOFT SET}

\subsection{Definition}

In this section we introduce the concept of picture fuzzy soft set This theory is a combination of our picture fuzzy set theory and the soft set theory was introduced by Molodtsov.

In [13] Molodtsov proposed a generalized tool for modeling complex systems involving uncertain or not clearly defined objects. Now we present a new definition, an example and some results which are the generalization of some propositions given in $[1,12]$.

Definition 5.1. Let $P F S(U)$ be the set of all picture fuzzy sets of $U$. Let $E$ be the set of parameters and $A \subseteq E$. A pair $(F, A)$ is called a picture fuzzy soft set over $U$, where $F$ is a mapping given by $F: A \rightarrow P F S(U)$.

Clearly, for any parameter $e \in E, F(e)$, can be written as a picture fuzzy set such that $F(e)=\left\{\left(x, \mu_{F(e)}(x), \eta_{F(e)}(x), \nu_{F(e)}(x)\right) \mid x \in U\right\}$, where the $\mu_{F(e)}(x)$ be the degree of positive membership, $\eta_{F(e)}(x)$ be the degree of neutral membership and $\nu_{F(e)}(x)$ be the degree of negative membership functions respectively. If for any parameter $e \in A$ and for any $x \in U, \eta_{F(e)}(x)=0$, then $F(e)$ will degenerated to be an intuitionistic fuzzy set and then $(F, A)$ is degenerated to be an intuitionistic fuzzy soft set. We denote the set of all picture fuzzy soft sets over $U$ by $P f s s(U)$.

Example 5.1. Consider a picture fuzzy soft set $(F, A)$, where $U$ is the set of four economic projects under the consideration of a decision committee to choose, which is denoted by $U=\left\{p_{1}, p_{2}, p_{3}, p_{4}\right\}$, and $A$ is a parameter set, where $A=\left\{e_{1}, e_{2}, e_{3}, e_{4}, e_{5}\right\}=\operatorname{good}$ finance indicator, average finance indicator, good social contribution, average social contribution, good environment indicator. The picture fuzzy soft set $(F, A)$ describes the attractiveness of the projects to the decision committee.

Suppose that

$$
\begin{gathered}
F\left(e_{1}\right)=\left\{\left(p_{1}, 0.8,0.12,0.05\right),\left(p_{2}, 0.6,0.18,0.16\right),\left(p_{3}, 0.55,0.20,0.21\right),\left(p_{4}, 0.50,0.20,0.24\right)\right\} \\
F\left(e_{2}\right)=\left\{\left(p_{1}, 0.82,0.05,0.10\right),\left(p_{2}, 0.7,0.12,0.10\right),\left(p_{3}, 0.60,0.14,0.10\right),\left(p_{4}, 0.51,0.10,0.24\right)\right\}, \\
F\left(e_{3}\right)=\left\{\left(p_{1}, 0.60,0.14,0.16\right),\left(p_{2}, 0.55,0.20,0.16\right),\left(p_{3}, 0.70,0.15,0.11\right),\left(p_{4}, 0.63,0.12,0.18\right)\right\}, \\
F\left(e_{4}\right)=\left\{\left(p_{1}, 0.7,0.12,0.07\right),\left(p_{2}, 0.75,0.05,0.16\right),\left(p_{3}, 0.60,0.17,0.18\right),\left(p_{4}, 0.55,0.10,0.22\right)\right\}, \\
F\left(e_{5}\right)=\left\{\left(p_{1}, 0.60,0.12,0.07\right),\left(p_{2}, 0.62,0.14,0.16\right),\left(p_{3}, 0.55,0.10,0.21\right),\left(p_{4}, 0.70,0.20,0.05\right)\right\} .
\end{gathered}
$$

The picture fuzzy soft set $(F, A)$ is a parameterized family $\left\{F\left(e_{i}\right): i=1,2,3,4,5\right\}$ of picture fuzzy sets over $U$.

\subsection{Some operations and properties}

Now we define some operations on picture fuzzy soft sets and present some properties. The other results of picture fuzzy soft sets could be seen in [9].

Definition 5.2. The complement of a picture fuzzy soft set $(F, A)$ is denoted as $(F, A)^{c}$ and is defined by $(F, A)^{c}=\left(F^{c}, A\right)$, where $F^{c}: A \rightarrow P F S(U)$ is a mapping given by $F^{c}(e)=(F(e))^{c}$, for all $e \in A$. 
Definition 5.3. If $(F, A)$ and $(G, B)$ are two picture fuzzy soft sets over a common universe $U$, then $(F, A)$ and $(G, B)$ is a picture fuzzy soft set denoted by $(F, A) \wedge(G, B)$ is defined by $(F, A) \wedge(G, B)=(H, A \times B)$, where $H(\alpha, \beta)=F(\alpha) \cap G(\beta)$ for all $(\alpha, \beta) \in A \times B$, that is

$$
\begin{aligned}
& H(\alpha, \beta)(x)=\left(\min \left(\mu_{F(\alpha)}(x), \mu_{G(\beta)}(x)\right), \min \left(\eta_{F(\alpha)}(x), \eta_{G(\beta)}(x)\right), \max \left(\nu_{F(\alpha)}(x), \nu_{G(\beta)}(x)\right)\right) \\
& \forall(\alpha, \beta) \in A \times B, \quad \forall x \in U
\end{aligned}
$$

Definition 5.4. If $(F, A)$ and $(G, B)$ are two picture fuzzy soft sets over a common universe $U$, then $(F, A)$ or $(G, B)$ is a picture fuzzy soft set denoted by $(F, A) \vee(G, B)$ is defined by $(F, A) \vee(G, B)=(H, A \times B)$, where $H(\alpha, \beta)=F(\alpha) \cup G(\beta)$ for all $(\alpha, \beta) \in A \times B$, that is

$$
\begin{aligned}
& H(\alpha, \beta)(x)=\left(\max \left(\mu_{F(\alpha)}(x), \mu_{G(\beta)}(x)\right), \min \left(\eta_{F(\alpha)}(x), \eta_{G(\beta)}(x)\right), \min \left(\nu_{F(\alpha)}(x), \nu_{G(\beta)}(x)\right)\right) \\
& \forall(\alpha, \beta) \in A \times B, \quad \forall x \in U
\end{aligned}
$$

Theorem 5.1. Let $(F, A)$ and $(G, B)$ be two picture fuzzy soft sets over $U 4$, then we have the following properties

(1) $((F, A) \wedge(G, B))^{c}=(F, A)^{c} \vee(G, B)^{c}$

(2) $((F, A) \vee(G, B))^{c}=(F, A)^{c} \wedge(G, B)^{c}$

Proof. See [9].

Definition 5.5. The intersection of two picture fuzzy soft sets $(F, A)$ and $(G, B)$ over a common universe $U$ is a picture fuzzy soft set $(H, C)$, where $C=A \cup B$ and for all $e \in C$,

$$
H(e)= \begin{cases}F(e) & \text { if } e \in A-B \\ G(e) & \text { if } e \in B-A \\ F(e) \cap G(e) & \text { if } e \in A \cap B\end{cases}
$$

Its mean

$H(e)=\left\{\left(x, \min \left(\mu_{F(e)}(x), \mu_{G(e)}(x)\right), \min \left(\eta_{F(e)}(x), \eta_{G(e)}(x)\right), \max \left(\nu_{F(e)}(x), \nu_{G(e)}(x)\right) \mid x \in U\right\}, \forall e \in A \cap B\right.$

This relation is denoted by $(F, A) \cap(G, B)=(H, C)$.

Definition 5.6. The union of two picture fuzzy soft sets $(F, A)$ and $(G, B)$ over a common universe $U$ is a picture fuzzy soft set $(H, C)$, where $C=A \cup B$ and for all $e \in C$,

$$
H(e)= \begin{cases}F(e) & \text { if } e \in A-B, \\ G(e) & \text { if } e \in B-A, \\ F(e) \cup G(e) & \text { if } e \in A \cap B .\end{cases}
$$

It's mean

$H(e)=\left\{\left(x, \max \left(\mu_{F(e)}(x), \mu_{G(e)}(x)\right), \min \left(\eta_{F(e)}(x), \eta_{G(e)}(x)\right), \min \left(\nu_{F(e)}(x), \nu_{G(e)}(x)\right) \mid x \in U\right\}, \forall e \in A \cap B\right.$

This relation is denoted by $(F, A) \cup(G, B)=(H, C)$ 
Theorem 5.2. Let $(F, A)$ and $(G, B)$ be two picture fuzzy soft sets over $U$, then we have the following properties:

(1) $((F, A) \cap(G, B))^{c}=(F, A)^{c} \cup(G, B)^{c}$

(2) $((F, A) \cup(G, B))^{c}=(F, A)^{c} \cap(G, B)^{c}$

Proof. See [9].

\section{AN APPLICATION}

In this section an application of PFS to a simple decision making problem should be presented. Suppose the following one criterion decision-making is considered:

Let $\mathcal{A}$ be a finite alternative set $\mathcal{A}=\left\{A_{1}, \cdots, A_{n}\right\}$. Suppose the basic evaluations of alternatives according to the criterion is given as a PFS $E$ on $\mathcal{A}$.

Let $E=\left\{e\left(A_{1}\right), \cdots, e\left(A_{n}\right)\right\}$, where for all $i, e\left(A_{i}\right)=\left(\mu\left(A_{i}\right), \eta\left(A_{i}\right), \nu\left(A_{i}\right)\right), 0 \leq \mu\left(A_{i}\right), \eta\left(A_{i}\right)$, $\nu\left(A_{i}\right) \leq 1, \mu\left(A_{i}\right)+\eta\left(A_{i}\right)+\nu\left(A_{i}\right) \leq 1$ The decision making problem is to rank the alternative set and to choose the best solution.

The following algorithm is used.

Algorithm 6.1.

Step 1. Define score functions on $\mathcal{A} h_{1}\left(A_{i}\right)=\mu\left(A_{i}\right),{ }_{i}, h_{2}\left(A_{i}\right)=\eta\left(A_{i}\right),{ }_{i}, h_{3}\left(A_{i}\right)=\mu\left(A_{i}\right)+$ $\eta\left(A_{i}\right)-\nu\left(A_{i}\right), \forall_{i}$.

\section{Step 2.}

Define order $\gtrsim_{1}$ on $\mathcal{A}$ by using $A_{i} \gtrsim_{1} A_{k}$ iff $h_{1}\left(A_{i}\right) \geq h_{1}\left(A_{k}\right)$,

Define order $\gtrsim_{2}$ on $\mathcal{A}$ by using $A_{i} \gtrsim_{2} A_{k}$ iff $h_{2}\left(A_{i}\right) \geq h_{2}\left(A_{k}\right)$,

Define order $\gtrsim_{3}$ on $\mathcal{A}$ by using $A_{i} \gtrsim_{3} A_{k}$ iff $h_{3}\left(A_{i}\right) \geq h_{3}\left(A_{k}\right)$,

Step 3. Define on $\mathcal{A}$ aggregation order $\gtrsim_{*}$ by using $\gtrsim_{1}, \gtrsim_{2}, \gtrsim_{3}$. and rank the alternative set and choose the solution.

\section{CONCLUSIONS}

In this paper, the new concept of picture fuzzy sets was introduced. Then some operations on PFSs and some properties of these operations were presented. Next, more new operations, including the convex combination of PFS, picture fuzzy relations with some their compositions were discussed. We also study the picture fuzzy soft set, and the Zadeh extention principle and a simple application of PFS to a one criterion decision making problem were presented. In the following paper some classes of aggregation operations of picture fuzzy information with application should be proposed.

\section{ACKNOWLEDGMENT}

This research is funded by the Vietnam National Foundation for Science and Technology Development (NAFOSTED) under grant numbers 102.01-2012.14 and 102.05.2014.01 


\section{REFERENCES}

[1] M. I. Ali, F. Feng, X. Liu, W. K. Min, and M. Shabir, "On some new operations in soft set theory," Computers \&3 Mathematics with Applications, vol. 57, no. 9, pp. 1547-1553, 2009.

[2] K. Atanassov and G. Gargov, "Interval valued intuitionistic fuzzy sets," Fuzzy sets and systems, vol. 31, no. 3, pp. 343-349, 1989.

[3] K. T. Atanassov, "Intuitionistic fuzzy sets," Fuzzy sets and Systems, vol. 20, no. 1, pp. 87-96, 1986.

[4] — On Intuitionistic Fuzzy Sets Theory. Springer, Berlin, Heidelberg, 2012.

[5] P. Burillo and H. Bustince, "Intuitionistic fuzzy relations. (part i)," Mathware 83 Soft Computing, vol. 2 , no. 1 , pp. 5-38, 2008.

[6] H. Bustince, "Construction of intuitionistic fuzzy relations with predetermined properties," Fuzzy Sets and Systems, vol. 109, no. 3, pp. 379-403, 2000.

[7] B. C. Cuong, "Picture fuzzy sets first results. part 1," Seminar Neuro-Fuzzy Systems with Applications, Preprint 03/2013, Institute of Mathematics, Hanoi, May 2013.

[8] — , "Picture fuzzy sets first results. part 2," Seminar Neuro-Fuzzy Systems with Applications, Preprint 04/2013, Institute of Mathematics, Hanoi, June 2013.

[9] B. C. Cuong and V. Kreinovich, "An extended soft set model: picture fuzzy soft set," Seminar Neuro-Fuzzy Systems with Applications, Preprint 04/2014, Institute of Mathematics, Hanoi, June 2014.

[10] —_ " "Picture fuzzy sets-a new concept for computational intelligence problems," 2013, Proceedings of the Third World Congress on Information and Communication Technologies WICT'2013, Hanoi, Vietnam, December 15-18, 2013, pp. 1-6.

[11] J. C. Fodor and M. Roubens, Fuzzy preference modelling and multicriteria decision support. Springer, 1994, vol. 14.

[12] P. Maji, R. Biswas, and A. R. Roy, "Soft set theory," Computers $\&$ Mathematics with Applications, vol. 45, no. 4, pp. 555-562, 2003.

[13] D. Molodtsov, "Soft set theoryfirst results," Computers $\mathcal{E}$ Mathematics with Applications, vol. 37, no. 4, pp. 19-31, 1999.

[14] H. Nguyen and E. Walker, A First Course in Fuzzy Logic. 2000, 2000.

[15] E. Szmidt and J. Kacprzyk, "Distances between intuitionistic fuzzy sets," Fuzzy sets and systems, vol. 114, no. 3, pp. 505-518, 2000.

[16] L. A. Zadeh, "Fuzzy sets," Information and control, vol. 8, no. 3, pp. 338-353, 1965.

Received on September 26 - 2014

Revised on October 07 - 2014 